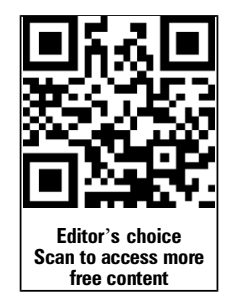

\title{
A prospective randomised controlled clinical trial comparing a combination of repeated intravitreal Ozurdex and macular laser therapy versus macular laser only in centre-involving diabetic macular oedema (OZLASE study)
}

\author{
Ling Zhi Heng, Sobha Sivaprasad, Roxanne Crosby-Nwaobi, Zubin Saihan, \\ Mike Karampelas, Catey Bunce, Tunde Peto, Philip G Hykin
}

Moorfields Eye Hospital NHS Foundation Trust, UCL Institute of Ophthalmology, NIHR Moorfields Biomedical Research Centre, London, UK

\section{Correspondence to}

Sobha Sivaprasad, Moorfields Eye Hospital NHS Foundation

Trust, UCL Institute of Ophthalmology, NIHR Moorfields Biomedical Research Centre, Moorfields Eye Hospital, 162, City Road, London EC1V 2PD, UK; senswathi@aol.com

Received 14 May 2015 Revised 14 September 2015 Accepted 19 September 2015 Published Online First 15 October 2015

\section{CrossMark}

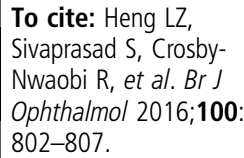

\section{ABSTRACT}

Background/aims To evaluate the clinical efficacy and safety of combined repeated Ozurdex and macular laser therapy (MLT) compared with MLT monotherapy in participants with visual impairment due to centreinvolving diabetic macular oedema (DMO).

Methods 80 patients with best corrected visual acuity (BCVA) between 54 and 78 ETDRS letters due to centreinvolving DMO were randomised to combination therapy with Ozurdex and MLT or MLT only. The combination arm received mandated Ozurdex injections at baseline and 16 weeks followed by retreatment criteria-guided pro-re-nata therapy at 32 and 48 weeks. Patients randomised to MLT only were treated every 16 weeks if clinically significant macular oedema was present. The primary outcome was the mean change from baseline in BCVA between arms at 56 weeks.

Results The mean change in BCVA at 56 weeks was -0.3 (SD 11.4) ETDRS letters in the combination arm versus +0.4 (SD 9.6) ETDRS (Early Treatment Diabetic Retinopathy study) letters in the MLT arm (effect estimate $1.15(95 \% \mathrm{Cl}-3.32$ to 5.61$))$. However, at 56 weeks, a post hoc comparison of central subfield thickness (CST) showed a decrease of $-113 \mu \mathrm{m}$ (IQR $-218,-64)$ (combination) versus $-17 \mu \mathrm{m}(-128,12)$ (MLT arm) $(p<0.001)$. Elevated intraocular pressure requiring topical therapy was observed in $8(20 \%)$ eyes in the combination versus $1(2.5 \%)$ in the MLT arm. $33 \%$ (9/27) of phakic patients in the combination arm underwent cataract surgery.

Conclusions Visual outcome following combination therapy did not differ from MLT alone in the centreinvolving DMO despite a significant decrease in CST likely due to an entry visual acuity-related ceiling effect and cataract development.

Trial registration number EudraCT 2011-003339-74

\section{INTRODUCTION}

Macular oedema is a frequent cause of impaired visual acuity in people with diabetes. During the last decade, studies on intravitreal inhibitors of vascular endothelial growth factor (VEGF) have demonstrated significant improvement in mean visual acuity in eyes with centre-involving diabetic macular oedema (DMO) superior to those achieved with macular laser therapy (MLT). ${ }^{1-5}$ However, optimal outcomes with these agents are only achieved if patients are treated repeatedly in the first year and monitored frequently to provide individualised retreatment thereafter. Despite intensive therapy, approximately $50 \%$ show persistence or recurrence of macular oedema at 12 months. ${ }^{6}$ For this reason, but also because in the early stages of DMO patients may be unwilling to commit to repeated injection and a single longer acting treatment may be attractive, other treatment options have been considered.

The rationale for corticosteroids to treat DMO is based on their broad range of actions. ${ }^{7}$ In addition to inhibition of VEGF expression, their antiinflammatory properties may attenuate the disease process. $^{8}{ }^{9}$ In 2009 , Ozurdex $0.7 \mathrm{mg}$, a sustained release dexamethasone drug delivery system showed clinical efficacy in macular oedema secondary to retinal vein occlusion and that single and repeated treatment with Ozurdex at 6 monthly intervals had a favourable safety profile over 12 months. ${ }^{10}$ The early PLACID study (dexamethasone intravitreal implant in combination with laser photocoagulation for the treatment of diffuse diabetic macular edema) compared 6 monthly Ozurdex versus MLT for DMO and showed no difference in the percentage of participants gaining $\geq 10$ letters best corrected visual acuity (BCVA) at 12 months. ${ }^{11}$ The phase III study of Ozurdex for DMO (MEAD) evaluated the 3 -year outcome of six monthly Ozurdex dosing based on prespecified retreatment criteria. It showed that the percentage of participants with $\geq 15$-letter improvement in BCVA at study end was greater with Ozurdex versus sham. ${ }^{12}$ Monthly monitoring of visual acuity and optical coherence tomography (OCT) central subfield thickness (CST) in patients with Ozurdex treated macular oedema suggests the clinical efficacy of this drug peaks at 1216 weeks. ${ }^{13}$ The BEVORDEX study (a randomized clinical trial of intravitreal bevacizumab versus intravitreal dexamethasone for diabetic macular edema) recently reported that pro re nata dosing of intravitreal bevacizumab every 4 weeks versus Ozurdex every 16 weeks in eyes with DMO showed similar rates of visual acuity improvement with superior anatomic outcomes and fewer injections in favour of the latter. ${ }^{14}$ It is therefore important to ascertain the risk-benefit ratio of frequent Ozurdex dosing in combination with MLT to translate the above observations into clinical practice. The OZLASE study was designed to evaluate the clinical 
efficacy and safety of 16 weekly Ozurdex dosing with deferred MLT compared with MLT only in patients with centre-involving DMO.

\section{MATERIALS AND METHODS}

The OZLASE study was a phase III, single-centre, prospective, randomised, active-controlled trial approved by the National Research Ethics Committee (REC reference:11/WM/0265), monitored by an independent data monitoring committee in which each patient signed informed consent. Patients were recruited based on the following inclusion and exclusion criteria;

Inclusion criteria:

1. subjects of either sex aged 18 years or over

2. diagnosis of diabetes mellitus (type 1 or type 2 )

3. BCVA in the study eye between 54 and 78 letters at $1 \mathrm{~m}$ (Snellen visual acuity 20/80-20/30) at baseline

4. On clinical exam, retinal thickening due to DMO involving the centre of the macula and OCT central subfield $>300 \mu$ (Spectralis SD-OCT, Heildelberg Engineering, Germany) at baseline

5. intraocular pressure $<30 \mathrm{~mm} \mathrm{Hg}$

6. visual acuity in fellow eye $\geq 2 / 60$

Exclusion criteria:

1. Macular ischaemia (foveal avascular zone (FAZ) $>1000 \mu \mathrm{m}$ in diameter OR severe perifoveal intercapillary loss on fundus fluorescein angiography).

2. Macular oedema is considered to be due to a cause other than DMO.

3. A coexistent ocular disease that, in the opinion of the investigator, visual acuity would not improve from resolution of the macular oedema.

4. An ocular condition is present (other than diabetes) that, in the opinion of the investigator, might affect macular oedema or alter visual acuity during the course of the study.

5. A substantial cataract that, in the opinion of the investigator, is likely to be decreasing visual acuity by three lines or more (ie, cataract would be reducing acuity to $6 / 12$ or worse if the eye was otherwise normal).

6. History of treatment for DMO at any time in the past 3 months (such as focal/grid macular photocoagulation, intravitreal or peribulbar corticosteroids, anti-VEGF drugs, or any other treatment) in the study eye.

7. History of panretinal scatter photocoagulation (PRP) within 3 months prior to randomisation in the study eye.

8. Anticipated need for PRP in the 6 months following randomisation in the study eye.

9. Proliferative diabetic retinopathy in the study eye.

10. Haemoglobin A1c $>11.0 \%$.

11. Uncontrolled glaucoma (in investigator's judgement).

One eye per patient was recruited to the trial. If both eyes of the patients met the eligibility criteria, the eye with the worse BCVA at baseline was enrolled.

The patients were randomised using a 1:1 allocation ratio into either the combination or MLT arms using computerised block randomisation stratified by baseline BCVA in the study eye (54-66 or 67-78 letters). The use of randomly varying block sizes ensured that allocation sequence was concealed from the randomising researcher.

The patients and clinical investigators were not masked to the treatment assignment. Masking was planned for the outcome assessors including optometrists, OCT technicians and reading centre graders.

\section{Treatment regimen}

The patients randomised to the combination arm received two mandated doses of Ozurdex at baseline and at week 16. Further retreatment occurred at 32 or 48 weeks with a combination of Ozurdex and MLT if retreatment criteria were met. The patients randomised to the MLT arm received laser therapy according to the modified Early Treatment Diabetic Retinopathy study (ETDRS) macular laser guidelines at screening and at 16, 32 or 48 weeks if the retreatment criteria were met. Combination arm retreatment criteria were: (1) CST of the study eye of more than $300 \mu \mathrm{m}$ on Spectralis SD-OCT and visual acuity of less than 89 ETDRS letters; or (2) CST of less than $300 \mu \mathrm{m}$ on Spectralis SD-OCT and visual acuity is less than 84 letters. The minimum interval between treatments was at least 16 weeks and additional laser photocoagulation was at the discretion of the investigator.

\section{Follow-up assessments}

Efficacy and safety variables were evaluated at study visits every 8 weeks. Assessments included ETDRS BCVA with refraction at screening, 8, 24, 40 and 56 weeks. OCT CST was performed at screening and at all follow-up visits.

\section{Outcome measures}

The prespecified primary efficacy evaluation was the difference in mean BCVA at week 56 measured as an ETDRS letter score in the intent-to-treat (ITT) population of all randomised patients after adjusting for baseline visual acuity.

Other key efficacy variables included changes in BCVA from baseline at week 24 and 40 , the proportion of patients with $\geq 10$ letter or $\geq 15$ letter gain or loss at 56 weeks, change in the scores of vision-related quality of life as assessed by VFQ-25 and health status (EQ-5D) from baseline, change in CST on Spectralis SD-OCT, the number of Ozurdex implants and the number of laser treatments.

Key safety variables included adverse events categorised into those related to the treatment in the study eye, disease progression in the study eye, adverse events in fellow eye and systemic adverse events. The known Ozurdex-related adverse events were broadly classified into raised intraocular pressure (IOP), cataract progression and cataract surgery, and device-related adverse events. Cataract surgery was planned in patients in either arm, if there was a $\geq 10$ letter decrease in BCVA due to cataract, as judged by the investigator before the 56 week exit visit. The change in the grade of retinopathy, greatest linear dimension and the area of the FAZ from baseline were evaluated on fourfield colour fundus photographs and fluorescein angiography, respectively, by masked evaluators at an independent reading centre.

\section{Statistical analyses}

The primary analyses of efficacy parameters were performed for the ITT population. A per protocol (PP) population, including only all randomised patients that had 12-month visual acuity data, was also conducted. A sensitivity analysis was performed to estimate the effect of cataract surgery done as PP and otherwise, for patients who underwent cataract surgery during the study. Missing BCVA data were imputed with the method of last observation carried forward. The sample size was based on a difference in mean BCVA from baseline to week 56 in the combination arm of +5 ETDRS letters and -1.0 in the control arm assuming a common SD of 9.0 using a two group $t$ test with a 0.50 two-sided significance level. A sample size of 36 patients in each treatment group was required for $80 \%$ power to detect a 
six letter difference in means. Forty patients in each arm were planned to allow for $10 \%$ dropout. Secondary statistical analyses were performed, with analysis of variance and Kruskal-Wallis tests for continuous data and $\chi^{2}$ tests for categorical data. For examination of changes from baseline in visual acuity for various subgroup analyses, 95\% CIs for the mean changes were provided. Presentations of continuous data included medians (IQR) instead of or in addition to means (SD). A post hoc exploratory analysis was also done to understand the change in mean visual acuity in pseudophakic eyes.

\section{RESULTS}

\section{Patient population and disposition}

A total of 80 patients were enrolled in the study and were randomised to combination treatment with the Ozurdex plus MLT or to MLT alone. Figure 1 shows the Consolidated Standards of Reporting Trials diagram for the study. Overall, 95\% (38/40) of patients in the combination arm and $97.5 \%$ (39/40) of patients in the MLT arm completed the study (table 1).

Baseline characteristics of enrolled patients are shown in table 2. There were no significant differences in any parameters between the two groups.

\section{Best corrected visual acuity}

The mean change in BCVA at 56 weeks was -0.3 (SD 11.4) ETDRS letters in the combination arm versus +0.4 (SD 9.5)

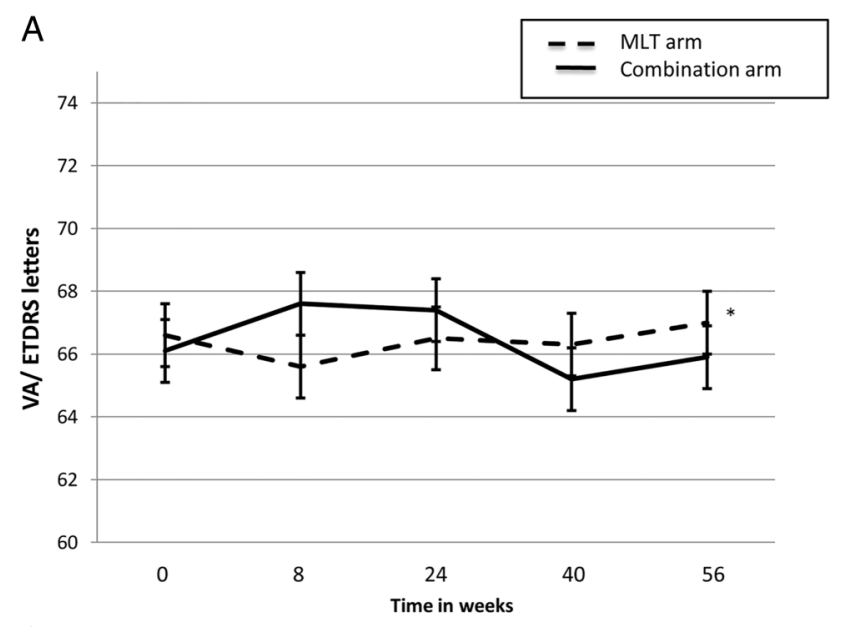

*error bars denote 1 standard deviation

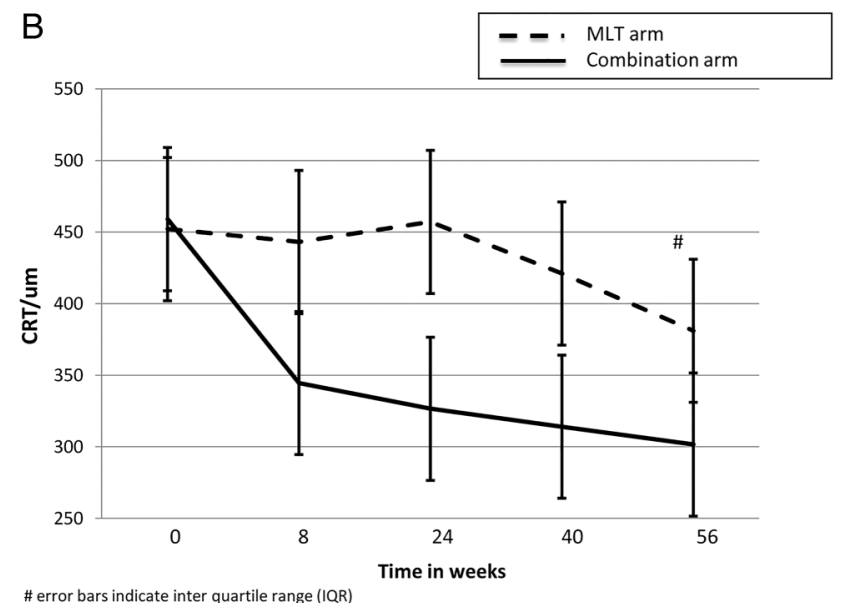

Figure 1 The OZLASE study: BCVA (A) and OCT CRT (B) versus time. BCVA, best-corrected visual acuity; CRT, central retinal thickness; MLT, macular laser therapy; OCT, optical coherence tomography.
Table 1 Baseline characteristics and patient disposition

\begin{tabular}{|c|c|c|}
\hline & $\begin{array}{l}\text { Ozurdex+laser } \\
\mathrm{N}=40\end{array}$ & $\begin{array}{l}\text { Laser } \\
\mathrm{N}=40\end{array}$ \\
\hline Number of patients (eyes), $n$ & 40 & 40 \\
\hline \multicolumn{3}{|l|}{ Sex, n (\%) } \\
\hline Males & $34(85.0)$ & $32(80.0)$ \\
\hline Females & $6(15.0)$ & $8(20.0)$ \\
\hline Age (years), mean (SD) & $65.6(10.6)$ & $61.1(12.8)$ \\
\hline \multicolumn{3}{|l|}{ Ethnicity, n (\%) } \\
\hline Asian or Asian British & $14(35.0)$ & $14(35.0)$ \\
\hline Black or Black British & 7 (17.5) & 7 (17.5) \\
\hline White or White British & $16(40.0)$ & $16(40.0)$ \\
\hline Other & $3(7.5)$ & $3(7.5)$ \\
\hline \multicolumn{3}{|l|}{ Diabetes, n (\%) } \\
\hline Type 1 & $2(5.0)$ & $4(10.0)$ \\
\hline Type 2 & $38(95.0)$ & $36(90.0)$ \\
\hline Duration of DM (years), median (IQR) & $15(11,20)$ & $15(9,22.5)$ \\
\hline HbA1c (\%), mean (SD) & $7.9(1.2)$ & $8.0(1.4)$ \\
\hline Systolic BP (mm Hg), mean (SD) & $130.7(16.6)$ & $130.8(16.0)$ \\
\hline Diastolic BP (mm Hg), mean (SD) & $72.0(9.4)$ & $76.1(9.2)$ \\
\hline ETDRS BCVA, mean (SD) & $66.1(7.3)$ & $66.6(7.7)$ \\
\hline \multicolumn{3}{|l|}{ Visual acuity group, $\mathrm{n}(\%)$} \\
\hline 54-66 letters & $22(55.0)$ & $21(52.5)$ \\
\hline $67-78$ letters & $18(45.0)$ & $19(47.5)^{*}$ \\
\hline $\begin{array}{l}\text { Duration of CSMO (months), median } \\
\text { (IQR) }\end{array}$ & $25.5(7.5,40.5)$ & $41(23.5,83.5)$ \\
\hline Number of prior MLTs, median (IQR) & $2(1,3)$ & $3(2,4)$ \\
\hline Prior anti-VEGF therapy & 2 & 2 \\
\hline Prior steroid therapy & None & 1 \\
\hline CST $(\mu \mathrm{m})$, median $(\mathrm{IQR})$ & $459(373,533)$ & $452(407.5,559)$ \\
\hline \multicolumn{3}{|l|}{ Lens status, n (\%) } \\
\hline Pseudophakic & $13(32.5)$ & $13(32.5)$ \\
\hline Phakic & $27(67.5)$ & $27(67.5)$ \\
\hline Previous treatment for glaucoma, $\mathrm{n}(\%)$ & $1(2.5)$ & $1(2.5)$ \\
\hline \multicolumn{3}{|c|}{$\begin{array}{l}\text { All percentages are out of N. } \\
\text { *One participant with } 81 \text { ETDRS letters was randomised in error. } \\
\text { BCA, best corrected visual acuity; BP, blood pressure; CST, central subfield thickness; } \\
\text { DM, diabetes mellitus; CSMO, clinically significant macular oedema; MLT, macular } \\
\text { laser therapy; } n \text {, number of participants/eyes; } N \text {, total number of participants/eyes; } \\
\text { VEGF, vascular endothelial growth factor. }\end{array}$} \\
\hline
\end{tabular}

ETDRS letters in the MLT arm (effect estimate 0.88 (95\% CI -3.46 to 5.22$)$ ). However, at 56 weeks, the CST decreased by $-113 \mu \mathrm{m} \quad(\mathrm{IQR}-218,-64)$ (combination) versus $-17 \mu \mathrm{m}$ $(-128,12) \quad$ (MLT arm) $(\mathrm{p}<0.001)$. Elevated IOP requiring topical therapy was observed in $8(20 \%)$ eyes in the combination versus $1(2.5 \%)$ in the MLT arm. Thirty-three per cent $(9 / 27)$ of phakic patients in the combination arm underwent cataract surgery. Table 2 shows the proportionate gain and losses of 10 or 15 ETDRS letters at 56 weeks and change in ETDRS letters at 24 and 40 weeks.

\section{Sensitivity analysis}

The sensitivity of the primary efficacy conclusions regarding cataract surgery was investigated, and the patients who underwent cataract surgery $(n=9)$ had marked mean visual acuity gain (effect size $+5.34[\mathrm{SD}=0.38,10.31]$ ) but this was insignificant for three patients who had undergone cataract surgery with $\leq 10$ letter loss prior to surgery (effect size 1.63, $\mathrm{p}=0.446$ ). Eight of the nine patients had a central retinal thickness (CRT) $>300 \mu \mathrm{m}$ prior to surgery. Post hoc analysis showed no significant differences in mean change in ETDRS BCVA between phakic $(-0.8$ [SD 12.5]) and pseudophakic eyes (+0.3 [SD 9.6]) (effect estimate $2.3(-4.8,9.3)$ in the combination arm. 
Table 2 Secondary endpoint analyses-efficacy outcome measures

\begin{tabular}{|c|c|c|c|c|c|c|}
\hline & $\begin{array}{l}\text { Ozurdex+laser } \\
\mathrm{N}=40\end{array}$ & $\begin{array}{l}\text { Number } \\
\text { missing }\end{array}$ & $\begin{array}{l}\text { Laser } \\
\mathrm{N}=40\end{array}$ & $\begin{array}{l}\text { Number } \\
\text { missing }\end{array}$ & $\begin{array}{l}\text { Effect } \\
\text { estimate }\end{array}$ & $95 \% \mathrm{Cl}$ \\
\hline Patients gaining $\geq 10$ ETDRS letters at 56 weeks from baseline, $n(\%)$ & $7(18.4)$ & 2 & $8(20.5)$ & 1 & 1.14 & 0.37 to 3.54 \\
\hline Patients gaining $\geq 15$ ETDRS letters at 56 weeks from baseline, $n(\%)$ & $6(15.8)$ & 2 & $2(5.1)$ & 1 & 0.29 & 0.05 to 1.53 \\
\hline Patients losing <15 ETDRS letters at 56 weeks from baseline, $\mathrm{n}(\%)$ & $35(92.1)$ & 2 & $35(89.7)$ & 1 & & \\
\hline Patients losing $\geq 30$ ETDRS letters at 56 weeks from baseline, $n(\%)$ & $1(2.6)$ & 2 & $0(0.0)$ & 1 & - & - \\
\hline Change in ETDRS BCVA at 24 weeks from baseline, mean (SD) & $1.3(8.8)$ & 4 & $-0.7(6.3)$ & 5 & -1.32 & -4.42 to 1.78 \\
\hline Change in ETDRS BCVA at 40 weeks from baseline, mean (SD) & $-1.1(12.8)$ & 8 & $-0.1(6.8)$ & 8 & 1.13 & -3.37 to 5.64 \\
\hline
\end{tabular}

All percentages are out of $\mathrm{N}$ minus number missing.

BCVA, best-corrected visual acuity; $n$, number of participants/eyes; $N$, total number of patients/eyes.

\section{Change in participant reported outcomes}

The mean change from baseline in domain scores of the NEI-VFQ 25 questionnaires was 1.6 (SD 10.4) in the combination arm and -0.4 (SD 12.2) in the MLT arm. There was also no difference in change in EQ-5D scores between arms.

\section{Change in macular thickness}

The median CST in the combination arm of $113 \mu \mathrm{m}$ (IQR $-218,-64)$ showed significant reduction compared with the MLT arm $(13 \mu \mathrm{m} \mathrm{IQR}-128,+12)$. The patients in both study groups showed a reduction of OCT-measured CST from baseline to week 56 (table 3). The steepest decline in CST in the combination arm was at 8 weeks.

\section{Number of treatments}

In the combination arm, the mean number of Ozurdex injections and MLT were 3.5 and 0.77 , respectively, and17 patients did not receive MLT, 15 received one and 8 received 2 treatments. In the MLT arm, the mean number of laser treatments was 3.05 .

\section{Change in ETDRS diabetic retinopathy severity score}

A higher proportion of patients improved their ETDRS diabetic retinopathy severity score by $\geq 1$ step in the combination arm $(14 / 40,35 \%)$ versus the MLT arm $(3 / 40,7.9 \%)$. At the end of the study, $54.3 \%$ in the combination versus $79 \%$ in the MLT arm remained at entry level and $5.8 \%$ versus $13.1 \%$, respectively, had worsened.

\section{Safety outcomes}

IOP

Elevated IOP requiring additional antiglaucoma medication occurred in $8(20 \%)$ eyes in the combination group versus 1 $(2.5 \%)$ in the MLT arm. No trabeculectomy or tube shunt surgery was required in either arm of the study.

\section{Cataract progression}

There were 27 phakic eyes in each arm. In the combination arm, 21/27 patients experienced cataract progression of whom 9 $(30 \%)$ had cataract surgery performed. In the laser arm, 4/27 subjects were noted to have progression of cataract but none underwent surgery in the study.

Other adverse events and serious adverse events

42 serious adverse events were reported during the study related primarily to hospitalisation for cataract surgery, or a systemic event for example, diabetic ketoacidosis. Two subjects in the laser arm suffered a stroke. Under-reporting of serious adverse events, progression of cataract and potential unmasking of optometrists were reported to the Medicines and Health Care Products Regulatory Authority, REC and to Allergan. There were no retinal tears, detachment or endophthalmitis in either arm. There were three device failures, but on each occasion, the device was easily removed from the sclera $(n=1)$ and subconjuctival space $(n=2)$ and a further device inserted.

\section{DISCUSSION}

The study demonstrated no difference in BCVA between the combination and MLT treatment arms at 56 weeks despite two mandated Ozurdex doses at 0 and 16 weeks and low threshold re-treatment criteria accounting for a mean of 3.5 Ozurdex injections in the combination arm. This result was unexpected in view of the visual gains experienced by patients after Ozurdex therapy in both the MEAD and PLACID studies between 4 and 12-week time-points, and there may be several explanations. First, it is possible that frequent dosing with Ozurdex does not provide additional visual function benefit compared with the less frequent dosing performed in MEAD and PLACID studies. However, the steady reduction in CST over the period of 56 weeks within OZLASE which differs to

Table 3 Post hoc analysis-CST at 8, 24 and 40 weeks

\begin{tabular}{lclll}
\hline & $\begin{array}{l}\text { Ozurdex+laser } \\
\text { N=40 }\end{array}$ & Number missing & $\begin{array}{l}\text { Laser } \\
\text { N=40 }\end{array}$ & Significance* \\
\hline CST at baseline, median (IQR) & $459(373$ to 533$)$ & 0 & $452(407.5$ to 559$)$ \\
CST at 8 weeks, median (IQR) & $344.5(302.5$ to 402$)$ & 0 & $443(398$ to 527$)$ \\
CST at 24 weeks, median (IQR) & $326.5(287$ to 387) & 4 & $457(363$ to 565$)$ \\
CST at 40 weeks, median (IQR) & $314(287$ to 387) & 8 & $421(360.5$ to 573$)$ \\
CST at 56 weeks, median (IQR) & $301.5(281$ to 379) & 2 & $381(339$ to 544$)$ & 8
\end{tabular}


the rise and trough effect on CST noted in the MEAD and PLACID studies argues against this. The lack of correlation between this anatomical improvement and the visual outcome suggests that the latter has been confounded by the development and progression of cataract following more frequent Ozurdex dosing, despite nine patients in the Ozurdex arm undergoing cataract surgery. Similar cataract-related confounding effects were noted in the MEAD study after the first year of follow up. Second, unlike other clinical trials investigating DMO therapy, this study only included patients with BCVA between 54 and 78 ETDRS letters. The rationale behind this limited inclusion criterion was to exclude patients with structural changes at the macula that were unlikely to respond to short-term therapy. However, as baseline visual acuity is negatively correlated with visual acuity gain after treatment, the study results may have been confounded by a ceiling effect on BCVA. ${ }^{3}$ Therefore, the potential for improvement in BCVA was less in this study compared with similar DMO studies, for example, the BEVORDEX and MEAD studies where visual acuity inclusion criteria were17-72 and 34-68 letters, respectively. ${ }^{11}{ }^{14}$ Furthermore, eight of nine patients undergoing cataract surgery had CRT $>300 \mu \mathrm{m}$ prior to surgery, which may have been exacerbated by the procedure itself limiting visual acuity gain. Finally, the PLACID study was a combination study of Ozurdex and MLT that failed to show superiority of this combination to MLT only. ${ }^{12}$ Although our study deferred MLT to 32 weeks in the combination arm, it did not have an Ozurdex monotherapy arm to evaluate the effect of withholding MLT completely, and it remains possible that the combination may have had an adverse effect on visual outcome.

Eyes that were pseudophakic at baseline did not demonstrate a visual benefit of combination therapy compared with phakic eyes despite a significant reduction in macular thickness in this arm. This may be explained by the small sample size $(n=12)$ and is similar to the negative subgroup analysis of a larger study that compared triamcinolone with MLT for DMO in 57 pseudophakic eyes. ${ }^{16}$ In contrast, the subgroup analysis of patients in the recent DRCR.net $(\mathrm{n}=124)^{3}{ }^{15}$ and Fluocinolone Acetonide in Diabetic Macular Edema (FAME) studies $(\mathrm{n}=141)^{17}$ showed superior effects with triamcinolone and fluocinolone acetonide inserts in the baseline pseudophakic cohorts, respectively. Other differences in patient characteristics in these studies may in part explain these conflicting results.

Eyes in the combination arm that underwent cataract surgery showed a significant improvement in BCVA at 56 weeks. Most cataract surgeries in the combination arm occurred between week 32 and 48 after exposure to between two and three intravitreal Ozurdex suggesting a dose-related cataract effect. This may explain why a higher proportion of patients showed cataract progression and surgery at 12 months in this study compared with the MEAD and PLACID studies where Ozurdex retreatment was less frequent.

We also observed that a significant proportion of patients showed a reduction in ETDRS diabetic retinopathy severity grade in the combination compared with the MLT arm. A similar effect to this anti-VEGF effect has been reported with fluocinolone acetonide and triamcinolone suggesting that steroids may promote regression of diabetic retinopathy as observed with anti-VEGF agents. ${ }^{17-19}$

Twenty per cent of combination arm patients experienced raised intraocular pressure requiring additional topical antiglaucoma treatment but no glaucoma surgery was necessary, and overall, the increased frequency of Ozurdex therapy did present significantly raised intraocular pressure concerns.
In conclusion, intensive 16 weekly retreatments with Ozurdex in eyes with centre-involving DMO did not result in better visual outcome in patients with baseline visual acuity between 58 and 74 letters despite a steady reduction in CST over 56 weeks likely related to a visual acuity ceiling effect and the development of cataract.

Contributors All authors have contributed as follows: Substantial contributions to the conception or design of the work, or the acquisition, analysis or interpretation of data. Drafting the work or revising it critically for important intellectual content. Final approval of the version published. Agreement to be accountable for all aspects of the work in ensuring that questions related to the accuracy or integrity of any part of the work are appropriately investigated and resolved.

Funding This investigator-initiated study was funded by Allergan International Ltd, Castlebar Road, Westport, County Mayo, Ireland (Allergan grant ref no: MAF-ISSOPH-RET-013). The research was supported by the National Institute for Health Research (NIHR) Biomedical Research Centre based at Moorfields Eye Hospital NHS Foundation Trust and UCL Institute of Ophthalmology. The views expressed are those of the author(s) and not necessarily those of the NHS, the NIHR or the Department of Health.

Competing interests PGH and SS have received research grants, travel grants and attended advisory board meetings of Novartis, Allergen and Bayer.

Ethics approval 11/WM/0265 West Midlands: Coventry and Warwickshire.

Provenance and peer review Not commissioned; externally peer reviewed.

Data sharing statement The database is available for further research on contacting the authors.

\section{REFERENCES}

1 Early Treatment Diabetic Retinopathy Study Research Group. Photocoagulation for diabetic macular edema. Early Treatment Diabetic Retinopathy Study report number 1. Arch Ophthalmol 1985;103:1796-806.

2 Early Treatment Diabetic Retinopathy Study Research Group. Early photocoagulation for diabetic retinopathy. ETDRS report number 9. Ophthalmology 1991;98:766-85.

3 Elman MJ, Aiello LP, Beck RW, et al., Diabetic Retinopathy Clinical Research Network. Randomized trial evaluating ranibizumab plus prompt or deferred laser or triamcinolone plus prompt laser for diabetic macular edema. Ophthalmology 2010;117:1064-77.

4 Michaelides $M$, Kaines $A$, Hamilton RD, et al. A prospective randomized trial of intravitreal bevacizumab or laser therapy in the management of diabetic macular edema (BOLT study) 12-month data: report 2. Ophthalmology 2010;117:1078-86.

5 Korobelnik JF, Do DV, Schmidt-Erfurth U, et al. Intravitreal aflibercept for diabetic macular edema. Ophthalmology 2014;121:2247-54.

6 Bressler SB, Qin H, Beck RW, et al., Diabetic Retinopathy Clinical Research Network. Factors associated with changes in visual acuity and central subfield thickness at 1 year after treatment for diabetic macular edema with ranibizumab. Arch Ophthalmol 2012;130:1153-61.

7 Augustin A, Loewenstein A, Kuppermann BD. Macular edema. General pathophysiology. Dev Ophthalmol 2010;47:10-26.

8 Sohn HJ, Han DH, Kim IT, et al. Changes in aqueous concentrations of various cytokines after intravitreal triamcinolone versus bevacizumab for diabetic macular edema. Am J Ophthalmol 2011;152:686-94.

9 Beck RW, Edwards AR, Aiello LP, et al., Diabetic Retinopathy Clinical Research Network (DRCR.net). Three-year follow-up of a randomized trial comparing focal/ grid photocoagulation and intravitreal triamcinolone for diabetic macular edema. Arch Ophthalmol 2009;127:245-51.

10 Haller JA, Bandello F, Belfort R Jr, et al., Ozurdex GENEVA Study Group. Dexamethasone intravitreal implant in patients with macular edema related to branch or central retinal vein occlusion twelve-month study results. Ophthalmology 2011;118:2453-60

11 Callanan DG, Gupta S, Boyer DS, et al. Dexamethasone intravitreal implant in combination with laser photocoagulation for the treatment of diffuse diabetic macular edema. Ophthalmology, 2013;120:1843-51.

12 Boyer DS, Yoon YH, Belfort R Jr, et al. Three-year, randomized, sham-controlled trial of dexamethasone intravitreal implant in patients with diabetic macular edema. Ophthalmology 2014;121:1904-14.

13 Mathew R, Pearce E, Muniraju R, et al. Monthly OCT monitoring of Ozurdex fo macular oedema related to retinal vascular diseases: re-treatment strategy (OCTOME Report 1). Eye (Lond) 2014:28:318-26.

14 Gillies MC, Lim LL, Campain A, et al. A Randomized clinical trial of intravitreal bevacizumab versus intravitreal dexamethasone for diabetic macular edema: the BEVORDEX study. Ophthalmology 2014;121:2473-81.

15 Elman MJ, Qin H, Aiello LP, et al., Diabetic Retinopathy Clinical Research Network. Intravitreal ranibizumab for diabetic macular edema with prompt versus deferred laser treatment: three-year randomized trial results. Ophthalmology 2012;119:2312-18. 
16 Diabetic Retinopathy Clinical Research Network. A randomized trial comparing intravitreal triamcinolone acetonide and focal/grid photocoagulation for diabetic macular edema. Ophthalmology 2008;115:1447-9.

17 Campochiaro PA, Brown DM, Pearson A, et al., FAME Study Group. Sustained delivery fluocinolone acetonide vitreous inserts provide benefit for at least 3 years in patients with diabetic macular edema. Ophthalmology 2012;119:2125-32.
18 Bressler SB, Qin H, Melia M, et al., Diabetic Retinopathy Clinical Research Network. Exploratory analysis of the effect of intravitreal ranibizumab or triamcinolone on worsening of diabetic retinopathy in a randomized clinical trial. JAMA Ophthalmol 2013;131:1033-40.

19 Ip MS, Domalpally A, Hopkins JJ, et al. Long-term effects of ranibizumab on diabetic retinopathy severity and progression. Arch Ophthalmol 2012;30:1145-52. 